\title{
APPLICATION OF AUTOMATON MODEL TO SUPPORT REHABILITATION OF MOTOR SKILLS OF PATIENTS
}

\author{
Grigorii Shovkoplias, Eduard Glekler, Vladislav Povyshev, Jaafar Hammoud, Natalia Gusarova, \\ Aleksandra Vatian and Anatoly Shalyto \\ ITMO University \\ Sankt-Petersburg 197101, Russia
}

\begin{abstract}
Rehabilitation of patients during illnesses associated with the loss of motor skills, such as multiple sclerosis, is an important task of medicine. Effective in these cases are gaming approaches that increase patient motivation, which in the field of e-Health form the direction of "Serious Games". The essential requirements in the development of Serious Games are the continuity of training, which should be carried out not only in a specialized center, but al-so by the patient himself at home, as well as the accuracy of the movements performed by the patient; otherwise, the outcome of the rehabilitation will be doubtful or even negative. As the basis for satisfying the rigorous requirements for the correctness of the movements performed the automatic simulation of the game process is chosen.

Serious Games differ from regular games in a significantly smaller number of possible states, which makes them convenient for applying this approach. At the same time, in contrast to the previously considered applications of au-tomaton models in medical systems, such as, for example, systems for sup-porting hemodialysis, in the case of a game, the parameters of each state can vary over a fairly wide range.

In the article, this problem is considered in the example of rehabilitation of pinch movements of hands by performing physical exercises in a specialized video game form using motion capture of fingers on a smartphone. The Ab-stract State Machine method is used to build the automaton model applicable to the Serious Game for pinch movement, which provides opportunities and motivation of the patient to do independent exercises. To build the resulting model, the method of successive refinements is used, on each of which veri-fication is performed, which provides the necessary control over the accuracy of patient movements.
\end{abstract}

\section{KEYWORDS}

Abstract State Machine, Serious Games, Rehabilitation Systems, Pinch Movements

\section{INTRODUCTION}

Rehabilitation of patients during illnesses associated with the loss of motor skills, such as multiple sclerosis, is an important task of medicine. Multiple sclerosis is a disease of the central nervous system, which may be accompanied by various neurological signs, including loss of motor skills. Radical treatments for multiple sclerosis have not yet been identified, and one of the most important factors in the rehabilitation of patients after exacerbations, as well as preventing or delaying the development of new exacerbations, is the regular exercise [NINDS, Halabchi]. But the patient's independent implementation of the complex of exercises is associated with motivational difficulties, as well as with the difficulty of assessing the correctness of the exercises without direct supervision of a specialist.

To increase the patient's motivation for regular exercise, a game approach is widely used, which is called in medical applications "serious games". In particular, in order to support the rehabilitation of patients with multiple sclerosis specialized video games using various motion capture technologies are offered [Haase, Jonsdottir, Midaglia]. However, most often the proposed video games require the use of special game consoles or specialized motion controllers, which can be difficult for the patient in his daily life. In addition, during rehabilitation through physical exercises, the correct execution of individual movement elements is important, which should be combined with a minimum restriction of patient freedom of movement. Otherwise, the exercises may not give the desired effect or even harm the patient. These contradictory requirements get critical when training in fine motor skills. The article discusses the possibilities of satisfying them by the example of developing serious games for training the pinch movement of the fingers. 


\section{BACKGROUND}

As the analysis showed, there are several categories of hand and finger motion controllers on the market that implement various motion capture technologies, including gloves with motion sensors like [CaptoGlove], Leap Motion controllers like [Leap], full-scale game controllers with six degrees of freedom like [Nintendo] and smartphones like [Xiaomi]. The first three categories allow you to fully capture all types of movements of the hands (the first two categories also allow you to fully capture all types of movements of the fingers), taking into account all degrees of freedom, while smartphones have the ability to fully capture the movements of the distal phalanges of 10 fingers simultaneously only in the plane of the smartphone screen.

However, smartphones, unlike other categories of devices, are gadgets of everyday use, they are familiar and convenient for the vast majority of patients. According to [Haase], approximately $85.6 \%$ of a sample of 570 patients with MS regularly use smartphones. Thus, the use of smartphones as a means of organizing regular rehabilitation exercises, inscribed in the daily life of the patient, and for motivational purposes is quite justified.

Consider the need and the possibility of organizing serious games when using a smartphone as a means of motion capture. From the point of view of modeling, most smartphone games for widespread use involve evaluation of the player's finger getting into a certain predetermined area of the screen, which, for example, can be a target or terminal state; however, tracking the current position of the finger outside this area is not required. At the same time, as mentioned above, when implementing rehabilitation exercises, the correct execution of individual movement elements is important. This opposition is confirmed in practice. According to [Jonsdottir], improvements in clinical indicators were detected in patients undergoing rehabilitation using specialized video games after 12 gaming sessions, while in patients playing active video games for a wide audience, no improvement was noted, although the patients themselves noted moral satisfaction from the process and subjective improvement in health status. This means that from the patient's point of view, the scenario of specialized games should be as close as possible to regular games, but at the same time keep all the advantages described above.

Thus, for the sterling rehabilitation of motor skills in patients with multiple sclerosis, it is necessary to develop specialized "serious games", and special requirements must be imposed on them:

- Automatic control and current monitoring of the correct execution of the patient's movements during the game, which will allow, on the one hand, to increase the effectiveness of the exercises, and on the other hand, to meaningfully interpret the results demonstrated by the patient without regular personal examination [Midaglia].

- Possibility of individual adaptation of a set of exercises and game scenario. For example, movements that are obviously impossible for a particular patient should be easily excluded from the scenario; otherwise, a deterioration in the state of health and demoralization of the patient may follow [Thirumalai].

To implement these requirements, it seems advisable to use the automaton model [Khoussainov, Shalyto, Polikarpova]. The concept of formal methods is widely used in healthcare (see review [Bonfanti]). As a rule, such applications as hemodialysis machine [Butler], infusion pump [Zhang], electrocardiography [Sobrinho], blood separator machine [Tolvanen] and other medical devices with a rigid and uniquely defined operation algorithm are considered. Respectively, the authors focus mainly on modeling, verification and validation of medical devices in accordance with pre-developed requirements. At the same time, the works related to the application of the automaton approach to the development of medical game applications ("serious games") were not found in the literature accessible to the authors.

Thus, the article sets the following tasks:

- Modeling hand and fingers movements in order to formulate requirements and constraints for the automaton model.

- Modeling the automaton that implements the identified requirements using abstract state machine tools.

- Offering an example of a scenario of a "serious game" for rehabilitation of motor skills restricted after multiple sclerosis based on a developed automaton model. 


\section{MODELING HAND AND FINGERS MOVEMENTS FOR REHABILITATION}

Pinch movement is the movement of the thumb and forefinger, the result of which is a pinch grip [Kapandji]. Pinch movement is the movement of the thumb and forefinger, the result of which is a pinch grip. From the point of view of patient rehabilitation, pinch grip, carried out by the pads of the thumb and index finger and allowing to hold relatively large objects, is of the greatest importance.

As a basis for assessing the correctness of pinch movement on a smartphone, the "Pinching Test" test was used from the set of active application tests for smartphones "FLOODLIGHT" [Midaglia]. This test consists in the fact that large round objects appear on the smartphone screen in front of the patient, which must be captured with a pinch movement. However, success is considered to be any closure of the thumb and forefinger, which does not always correspond to the anatomically correct pinch movement. In particular, our experiments showed that when the distal phalanges of the thumb and forefinger came together at an angle of $90^{\circ}$, the FLOODLIGHT application counted this movement as "success", although in this case the capture of a real object is fundamentally impossible.

In this regard, based on the anatomical description of the movements of the phalanges of the fingers during pinch grip, we built its mathematical (skeleton) model, which is illustrated in Figure 1.

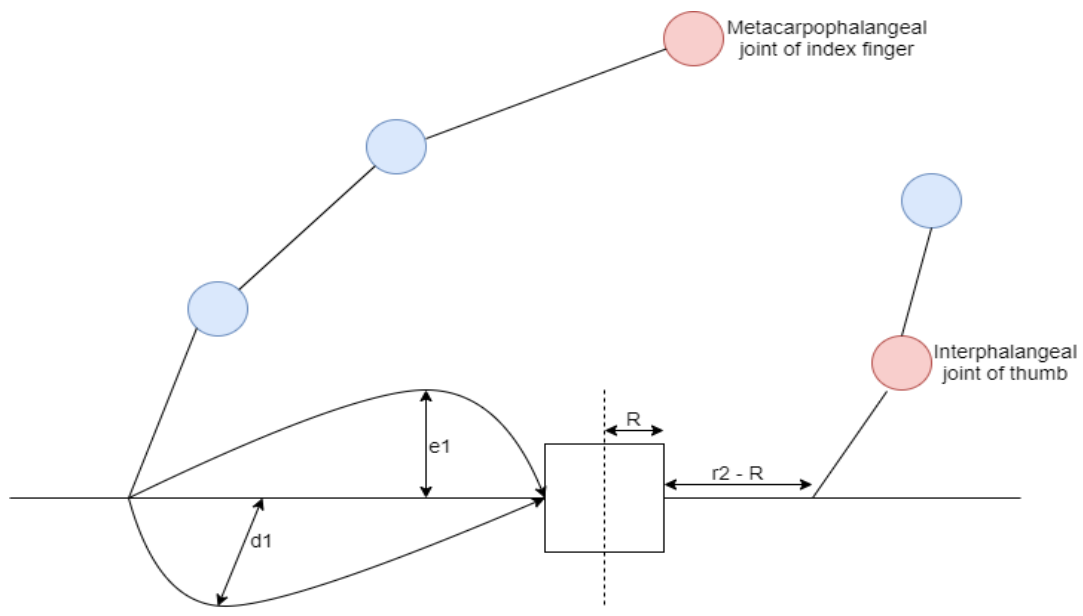

Figure 1. A skeleton model of pinch movement

The pinch movement begins with the location of the distal phalanges of the thumb and forefinger so that the center of the captured object is on a straight line with the corresponding phalanges. Moreover, hereafter, the center of the captured object is understood to be not necessarily the geometric center of the object, but a certain point near which the person wants to grip the given object. Next, the flexion movement of the metacarpophalangeal joint of the index finger occurs. The remaining joints at this time are almost motionless. A slight flexion movement of the distal interphalangeal joint of the thumb is acceptable. As a result of the bending of these joints, the distal phalanges of the index and thumb are approached at distances to the center of the object $\mathrm{r} 1$ and $\mathrm{r} 2$. The condition for the end of the movement is $\max (\mathrm{r} 1, \mathrm{r} 2) \leq \mathrm{R}$, where $\mathrm{R}$ is the distance from the center of the griped object to its edge. The distal phalanges of the thumb and forefinger approach each other in a straight line on which lies the center of the captured object, while the orthogonal components of the phalangeal shift vector $\mathrm{d}$ and e orthogonal to this straight line should not exceed $\mathrm{D}$ (in the plane orthogonal to the distal phalanx of the thumb) and $\mathrm{E}$ (in the plane, in which lies the distal phalanx of the thumb).

From the point of view of the effectiveness of rehabilitation, it is also necessary to introduce a restriction $\mathrm{T}$ on the duration $\mathrm{t}$ of the movement, i.e. $\mathrm{t} \leq \mathrm{T}$, as well as the number of repetitions of the pinch movement in one game session $\mathrm{m}$ with the restriction $\mathrm{M}$, i.e. $\mathrm{m} \leq \mathrm{M}$.

The control of pinch movement begins only from the moment the distal phalanges of the fingers are placed in a position on a straight line with the captured object (with a deviation b not exceeding the value of B, otherwise the movement is not considered erroneous, but it does not begin to be measured). The parameter 
$\mathrm{n}=\mathrm{r} 1 / \mathrm{r} 2$ is measured at the moment of the beginning of the movement and must be outside the norm $\mathrm{N}(\mathrm{N} \geq 1)$, otherwise the movement is immediately recognized as erroneous. Parameters $\mathrm{d}$ and e are measured throughout the movement; they must be within the limits of the norms $\mathrm{D}$ and $\mathrm{E}(\mathrm{d} \leq \mathrm{D}, \mathrm{e} \leq \mathrm{E}$, for both distal phalanges) and remain within these limits at any stage of the movement, otherwise it is immediately recognized as erroneous. The movement is considered complete when the parameters $\mathrm{r} 1$ and $\mathrm{r} 2$ are within the norm $\mathrm{R}$, if the time limit T has not been exceeded.

Thus, going beyond any of the seven parameters described above ( $\mathrm{n}, \mathrm{d}$ and e for the thumb and forefinger, $\mathrm{t}, \mathrm{m}$ ) is considered as an error in the pinch movement. Let us explain the anatomical meaning of these parameters. In Figure 1, the joints that should be active or accordingly inactive during the pinch movement are marked in red and blue. Going beyond the parameters d and e means that there has been a flexion of the joints, which should be inactive, or the movement of the hand or arm as a whole. It may also mean that there was a flexion of the joints involved in the movement, but not in that plane. Going beyond the parameter $n$ means that the distal phalanges were initially set incorrectly (for the correct movement, the distal phalanx of the thumb should be closer to the captured object).

In a meaningful sense, going beyond the specified parameters means errors in the implementation of rehabilitation movements and requires a statistical analysis with the subsequent involvement of a doctor for their interpretation and correction.

When implementing pinch movement on the smartphone screen, the distal phalanges of both fingers should be placed on the screen and then during the movement not to tear themselves away from it, i.e. in the above model, $\mathrm{E}=0$. Figure 2 shows the red trajectories of the distal phalanges on the screen in red, the black circle indicates the captured round object on the screen. After both distal phalanges have been placed on the screen, the distance $b$ is measured from the line between them to the center of the captured circle on the screen. If it is less than $\mathrm{B}$, the movement is considered to have begun. This limitation prevents accidental clicks. Next, there is a parallel transfer of the line between the distal phalanges to the point of the center of the circle. Subsequently, the deviation $\mathrm{d}$ for both phalanges (for each separately) will be calculated from this straight line. $\mathrm{r} 1$ and $\mathrm{r} 2$ are also counted from the projection of the coordinates of the phalanges onto this line.

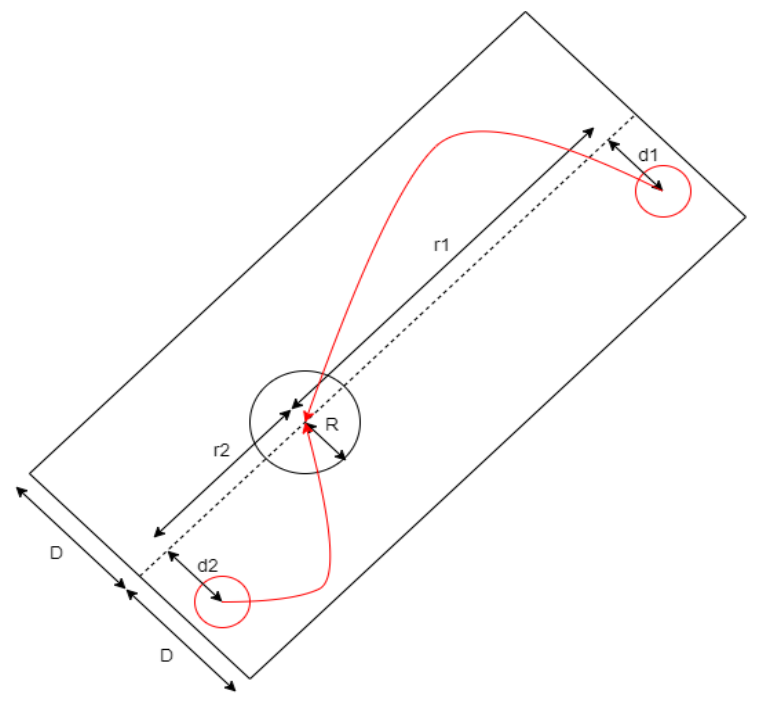

Figure 2. Trajectories of distal phalanges and their parameters

Based on the developed description of hand and fingers movements, we conclude that the game scenario can be a single sequence of states, but with variable parameters that are controls. Consequently, we can formulate requirements for the automaton model of "Serious Game" for pinch movement rehabilitation:

- $\quad$ it is required to provide the ability to change parameters directly during the game, i.e. after several iterations;

- $\quad$ statistics are required for each parameter separately. 


\section{AUTOMATA MODELING}

In order to build the automaton model we use the Abstract State Machine (ASM) method. The ASM for the game is built using the refining method as a sequential forming of the ground model, and then two levels of refinement. The ground model contains the basis of the test, not taking into account the errors that the patient may make during the capture procedure. The first refining includes the processing and collection of statistics for errors. The second refining adds the ability to the doctor to adapt the game scenario for a specific patient, based on the collected data. A detailed description of each stage of refinement is given below.

Ground model (Fig. 3). After the "Start of the session" (Initial state), the procedure for capturing the item occurs, according to the scenario. After M successful capture attempts, the game ends in the "End of the session" state. In the ground model, parameter $\mathrm{b}$ is measured and used to prevent accidental clicks. The r1 and r2 parameters are used to determine the condition for successful completion of the attempt. The parameters n, $\mathrm{d} 1, \mathrm{~d} 2$, and $\mathrm{t}$ are not used at this stage, since there is no separate collection of statistics for these parameters.

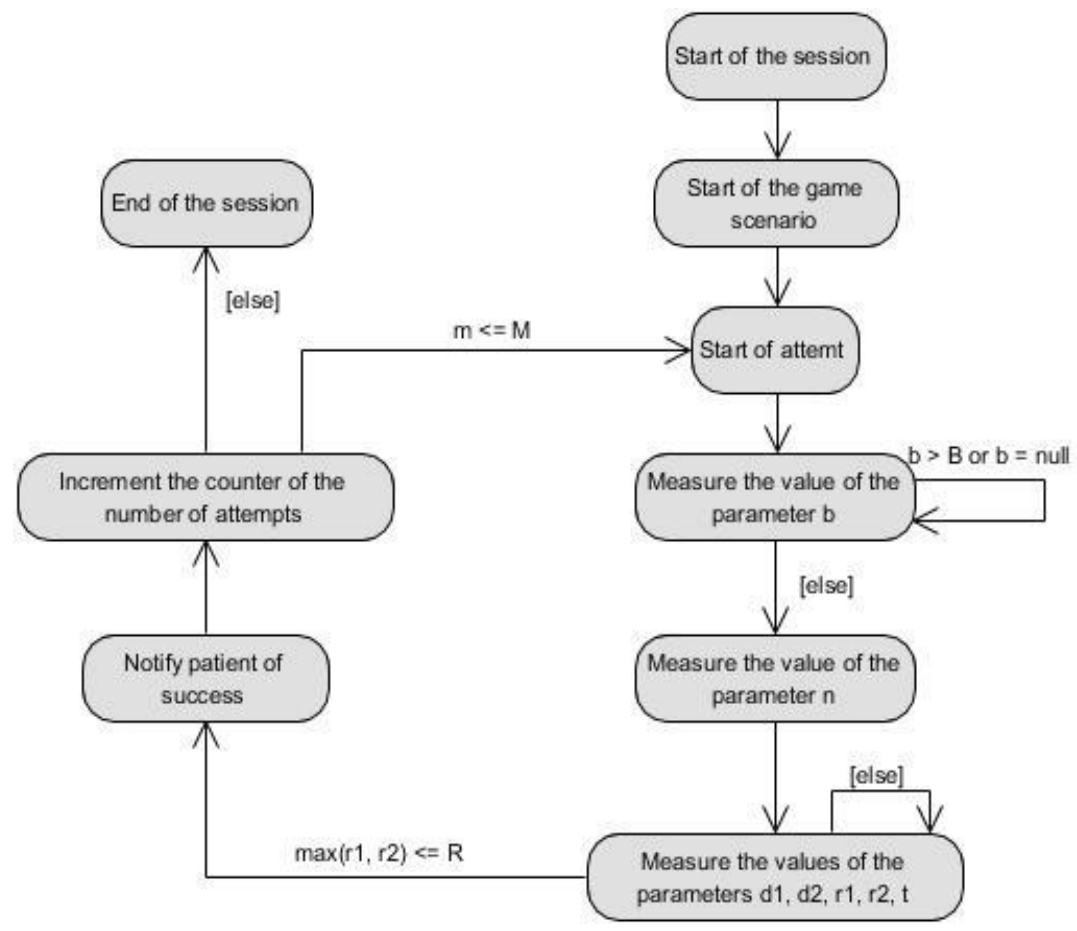

Figure 3. Ground model of automata

First refinement (Figure 4). As described earlier, when making a capture, various errors in hand movements can be made, which will be reflected in the parameters n, d1, d2 and t. All the described deviations are orthogonal and independent of each other, therefore, they can be added to the model in the same way except for the parameter $\mathrm{n}$, which is checked at an earlier stage - when placing fingers on the screen. In the framework of the same refining, the ability to collect statistics was added so that the doctor could later analyze it; for this, several states were included in the model that allow counting the number of successful and erroneous attempts (errors are calculated separately for each parameter).

Second refinement (Figure 5). In order for the doctor to adapt the game scenario, it is necessary to transfer information from the device to him and give him the opportunity to change the constants $\mathrm{R}, \mathrm{D}, \mathrm{N}, \mathrm{T}$ and $\mathrm{M}$, then, by the decision of the doctor, the game either continues or is stopped by transitions to the start one, or to final state.

Thus, the automaton modeling process for a serious game under consideration is described. The automaton model satisfies the above requirements, providing the ability to control and adapt constants affecting the game scenario. After each step, the automaton can be verified by standard means. 


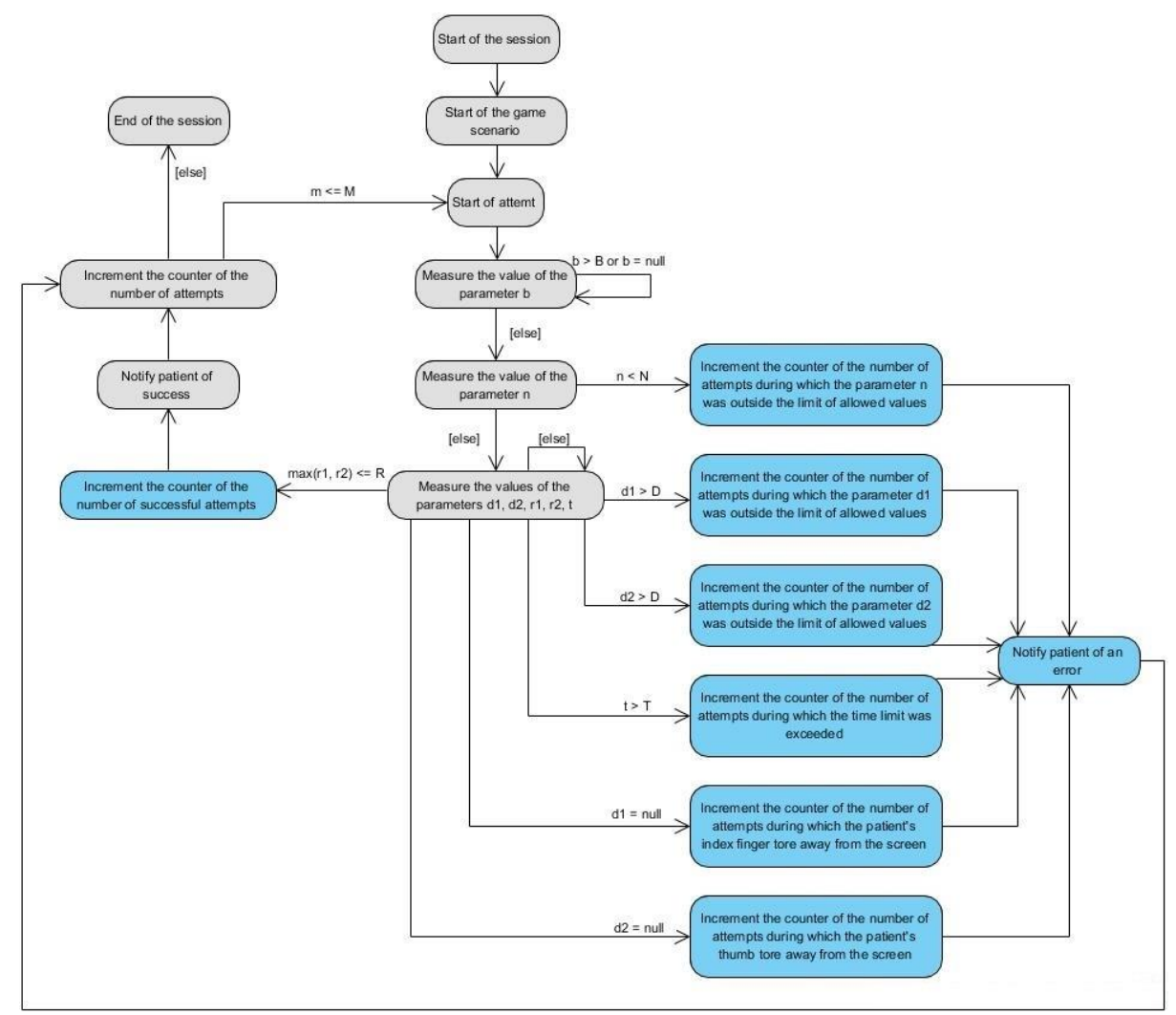

Figure 4. First refinement of automata

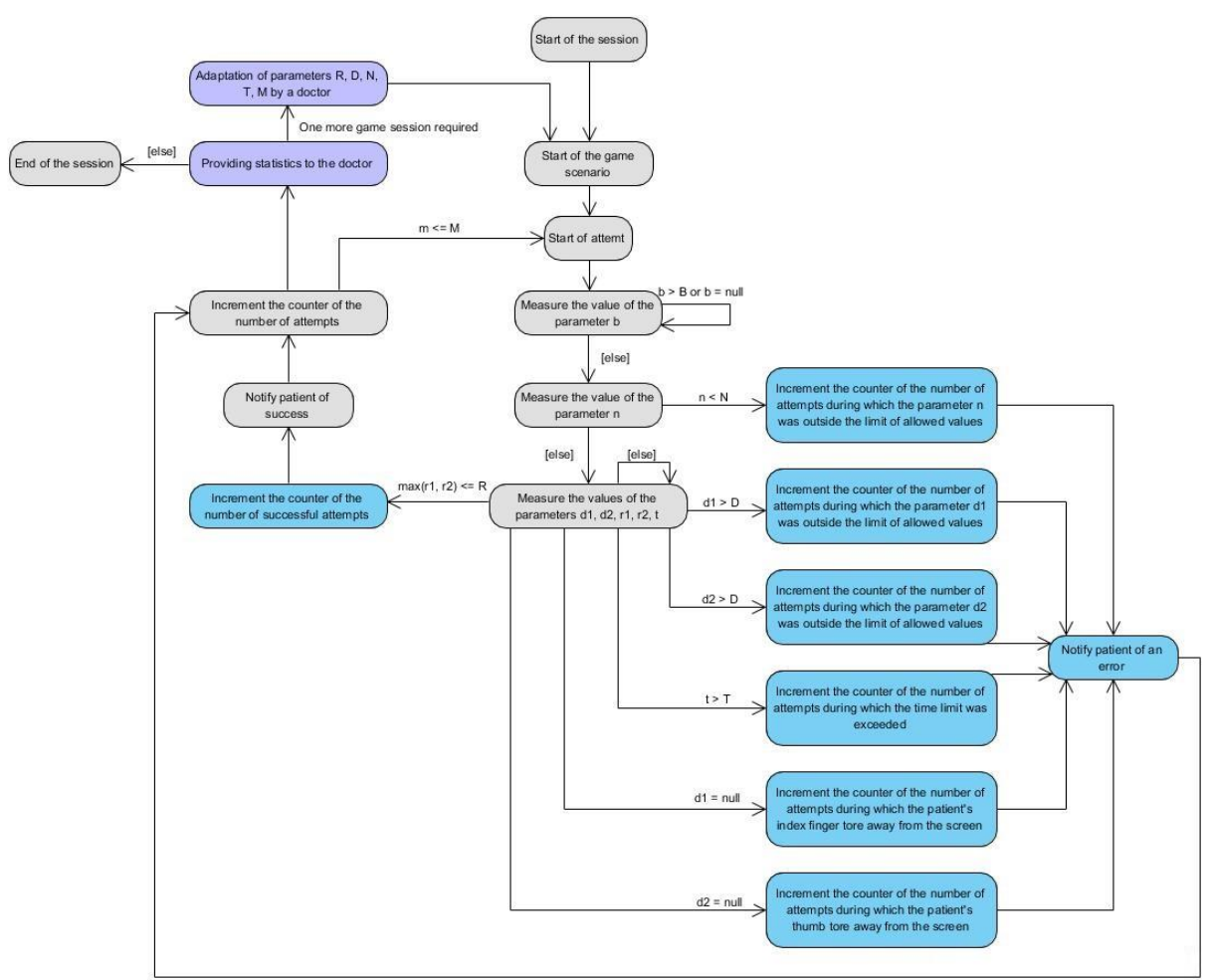

Figure 5. Second refinement of automata 


\section{5. "SERIOUS GAME" SCENARIO FOR REHABILITATION OF PINCH MOVEMENT}

The game should provide the patient with several attempts to pinch movement in an intuitive game form, in accordance with the previously established requirement for the involvement and motivation of patients.

An example of a possible test scenario is given below.

Before starting the game, the patient is explained his task, namely: to squeeze round objects appearing on the smartphone screen with the thumb and forefinger at a pace accessible to him, but preferably as soon as possible.

Next, the first round object appears on the screen in a random position. After the patient has set the distal phalanges in the correct starting position and started pinching movements, the object on the screen visually shrinks until the movement completes successfully - in this case, the object disappears. If the movement is made with an error, then the item on the screen restores its original shape. Thus, the patient is provided with intuitive visual information about whether he is moving correctly.

If the plucking attempt was completed with an error, the patient is offered a new attempt to capture the same object. Upon successful completion, the captured item disappears and a new one appears on the screen in a random position. The result of any attempt (successful or erroneous, taking into account the type of error) is recorded. The patient is offered $\mathrm{M}$ attempts in one session of the game. After the session is completed, the final results are generated, which counts the number of successes and mistakes made by the patient during the session, with each type of error taken into account separately. The results can be provided to a specialist doctor who, on their basis, can adjust the limitations (R, D, N, T and M) taking into account the characteristics of this patient for the next session.

It is easy to see that the developed game scenario satisfies the requirements formulated in Section 2: it adapts to the capabilities and needs of the patient, and at the same time, it contains variable parameters that are controls.

\section{CONCLUSION}

The article discusses the problem of rehabilitation of patients during illnesses associated with the loss of motor skills, such as multiple sclerosis. Effective in these cases are gaming approaches that increase patient motivation - so called "Serious Games".

Serious Games differ from regular games in a significantly smaller number of possible states, which makes them convenient for applying this approach. At the same time, in contrast to the previously considered applications of automaton models in medical systems, such as, for example, systems for supporting hemodialysis, in the case of a game, the parameters of each state can vary over a fairly wide range.

In the article, this problem is considered in the example of rehabilitation of pinch movements of hands by performing physical exercises in a specialized video game form using motion capture of fingers on a smartphone. The Abstract State Machine method is used to build the automaton model applicable to the Serious Game for pinch movement, which provides opportunities and motivation of the patient to do independent exercises. To build the resulting model, the method of successive refinements is used, on each of which verification is performed, which provides the necessary control over the accuracy of patient movements.

Thus, all the tasks formulated by the authors in the article are solved, namely:

- The model of hand and fingers movements is developed, that allowed us to formulate requirements and constraints for the automaton model.

- Using abstract state machine tools modeling the automaton was fulfilled that implements the identified requirements.

- Based on a developed automaton model an example of a scenario of a "serious game" for rehabilitation of motor skills restricted after multiple sclerosis is offered.

The developed approach seems quite general and can be extended to more complex and promising motion controllers, including those that support 3D movements, as well as to other types of hand movements. The authors consider such extensions as directions for their future work. 


\section{ACKNOWLEDGEMENT}

This work was financially supported by Russian Science Foundation, Grant 19-19-00696.

\section{REFERENCES}

Bonfanti S., Gargantini A., Mashkoor A. A Systematic Literature Review of the Use of Formal Methods in Medical Software Systems. J. Softw. Evol. and Proc. 00:1-23 (2017).

Butler MJ, Schewe K, Mashkoor A, Bir'o M ((eds.)). Abstract State Machines, Alloy, B, TLA, VDM, and Z - $5^{\text {th }}$ International Conference, ABZ 2016, Linz, Austria, May 23-27, 2016, Proceedings, Lecture Notes in Computer Science, vol. 9675, Springer, 2016, doi: 10.1007/978-3-319-33600-8. URL https://doi.org/10.1007/ 978-3-319-336008.

CaptoGlove applications. https://www.captoglove.com/features/, last access 2020/12/01

Haase R, Schultheiss T, Kempcke R, Thomas K, Ziemssen T. Use and Acceptance of Electronic Communication by Patients With Multiple Sclerosis: A Multicenter Questionnaire Study. J Med Internet Res 2012;14(5): e135

Halabchi F., Alizadeh Z., Sahraian M. A. \& Abolhasani M. Exercise prescription for patients with multiple sclerosis; potential benefits and practical recommendations. BMC Neurology, V.17, Article number: 185 (2017)

Jonsdottir J., Bertoni R., Lawo M., Montesano A. Serious Games for Arm Rehabilitation of Persons with Multiple Sclerosis. A randomized controlled pilot study. Multiple Sclerosis and Related Disorders 19, 25-29 (2018).

Kapandji I.A. The Physiology of the Joints, Volume 1: Upper Limb. 6th ed. Churchill Livingstone (2007).

Khoussainov B., Nerode A. Automata Theory and its Applications. Progress in Computer Science and Applied Logic, Volume 21. Birkhauser, 2001.

Leap Motion Controller. https://www.leapmotion.com/technology/, last access 2020/12/01

Midaglia L., Mulero P., Montalban X. et al. Adherence and Satisfaction of Smartphone- and Smartwatch-Based Remote Active Testing and Passive Monitoring in People With Multiple Sclerosis: Nonrandomized Interventional Feasibility Study. J Med Internet Res.;21(8): e14863 (2019). DOI: 10.2196/14863.

NINDS Multiple Sclerosis Information Page. National Institute of Neurological Disorders and Stroke. 18 Oct 2019. http://www.ninds.nih.gov/disorders/multiple_sclerosis/multiple_sclerosis.htm, last access 12.01.2020

Nintendo Wii. https://www.nintendo.ru/Wii/Wii-94559.html, last access 2020/12/01

Polikarpova N. I., Shalyto A. A. Automata-based programming. SPb.: Piter. 2009 (rus)

Shalyto A.A. Software Automation Design: Algorithmization and Programming of Problems of Logical Control. Journal of Computer and Systems Sciences International. 2000. Vol.39. №6, p.899-916.

Sobrinho, Cunha P, Da Silva L, Perkusich A, Cordeiro T, R^ego J. A simulation approach to certify electrocardiography devices. International Conference on E-health Networking, Application Services (HealthCom), Boston, MA, USA, 2015; 86-90, doi: 10.1109/HealthCom.2015.7454478.

Thirumalai M, Rimmer JH, Johnson G, Wilroy J, Young HJ, Mehta T, Lai B. TEAMS (Tele-Exercise and Multiple Sclerosis), a Tailored Telerehabilitation mHealth App: Participant-Centered Development and Usability Study. JMIR Mhealth Uhealth. 6(5): e10181(2018). DOI: 10.2196/10181

Tolvanen JP, Djuki'c V, Popovic A. Metamodeling for medical devices: Code generation, model-debugging and run-time synchronization. Procedia Computer Science, vol. 63, 2015; 539-544, doi: 10.1016/j.procs.2015.08.382.

Xiaomi Redmi Note 8 Pro. https://www.mi.com/ru/redmi-note-8-pro/, last access 2020/12/01

Zhang Y, Jetley R, Jones PL, Ray A. Generic Safety Requirements for Developing Safe Insulin Pump Software. Journal of Diabetes Science and Technology Nov 2011; 5(6):1403-1419. URL http://www.ncbi.nlm. nih.gov/pmc/articles/PMC3262707/, 00008. 